TRANSACTIONS OF THE

AMERICAN MATHEMATICAL SOCIETY

Volume 363, Number 5, May 2011, Pages 2467-2479

S 0002-9947(2010)04994-3

Article electronically published on December 7, 2010

\title{
ON QUASICONFORMAL MAPS WITH IDENTITY BOUNDARY VALUES
}

\author{
V. MANOJLOVIĆ AND M. VUORINEN
}

\begin{abstract}
Quasiconformal homeomorphisms of the unit ball $B^{n}$ of $\mathbb{R}^{n}, n \geq 3$, onto itself with identity boundary values are studied. A spatial analogue of Teichmüller's theorem is proved.
\end{abstract}

\section{INTRODUCTION}

For a domain $G \subset \mathbb{R}^{n}, n \geqslant 2$, let

$$
I d(\partial G)=\left\{f: \overline{\mathbb{R}^{n}} \rightarrow \overline{\mathbb{R}^{n}} \text { homeomorphism }: f(x)=x, \quad \forall x \in \overline{\mathbb{R}^{n}} \backslash G\right\} .
$$

Here $\overline{\mathbb{R}^{n}}$ stands for the Möbius space $\mathbb{R}^{n} \cup\{\infty\}$. We shall always assume that $\operatorname{card}\left\{\overline{\mathbb{R}^{n}} \backslash G\right\} \geq 3$. If $K \geqslant 1$, then the class of $K$-quasiconformal maps in $\operatorname{Id}(\partial G)$ is denoted by $I d_{K}(\partial G)$. Throughout this paper we adopt the standard notation and terminology from Väisälä's book [V]. In particular, $K$-quasiconformal maps are defined in terms of the maximal dilatation as in [V] p. 42] if not otherwise stated. The maximal dilatation of a homeomorphism $f: G \rightarrow G^{\prime}$ where $G, G^{\prime} \subset \mathbb{R}^{n}$ are domains, is denoted by $K(f)$.

The subject of this research is to study the following well-known problem.

1.1. Problem. (1) Given $a, b \in G$ and $f \in I d(\partial G)$ with $f(a)=b$, find a lower bound for $K(f)$.

(2) Given $a, b \in G$, construct $f \in \operatorname{Id}(\partial G)$ with $f(a)=b$ and give an upper bound for $K(f)$.

O. Teichmüller studied this problem in the case when $G$ is a plane domain with $\operatorname{card}\left(\overline{\mathbb{R}^{2}} \backslash G\right)=3$ and solved it by proving the following theorem with a sharp bound for $K(f)$.

1.2. Theorem. Let $G=\mathbb{R}^{2} \backslash\{0,1\}, a, b \in G$. Then there exists $f \in I d_{K}(\partial G)$ with $f(a)=b$ iff

$$
\log (K(f)) \geqslant s_{G}(a, b)
$$

where $s_{G}(a, b)$ is the hyperbolic metric of $G$.

Received by the editors May 12, 2008 and, in revised form, December 29, 2008.

2010 Mathematics Subject Classification. Primary 30C65; Secondary 30C62.

(C)2010 American Mathematical Society

Reverts to public domain 28 years from publication 
Motivated by a question of F.W. Gehring, J. Krzyż [K, Theorem 1] proved the following theorem. See also Teichmüller [T] and Krushkal [Kr, p. 59]. Write $B^{n}(r)=\left\{x \in \mathbb{R}^{n}:|x|<r\right\}$ and $B^{n}=B^{n}(1)$.

1.3. Theorem (Krzyż $\left[\mathrm{K}\right.$, Theorem 1]). For $f \in I d_{K}\left(\partial B^{2}\right)$ the sharp bounds are

$$
|f(0)| \leqslant \mu^{-1}\left(\log \frac{\sqrt{K}+1}{\sqrt{K}-1}\right) \equiv c_{1}
$$

where $\mu$ is the function defined in (2.4) and

$$
\tanh \frac{\rho_{B^{2}}(f(z), z)}{2} \leqslant c_{1}
$$

for every $z \in B^{2}$, where $\rho_{B^{2}}$ is the hyperbolic metric defined in Lemma 2.1 .

The constant $c_{1}$ in (1.4) is quite involved. It is hard to see how it behaves in the crucial passage to limit $K \rightarrow 1$. Therefore we give an explicit bound for this constant.

1.6. Lemma. The constant $c_{1}$ in (1.4) satisfies for $K>1$,

$$
\frac{K-1}{K+1}<c_{1}<2 \frac{K-1}{\sqrt{K}+1} .
$$

Later studies of this topic include the paper of G. Martin [M]. He formulated a question of the same type as Gehring did, but for general plane domains. This question was solved in the negative, at the same time by A. Solynin and M. Vuorinen [SV] and H. Xinzhong and N.E. Cho [XC].

Our goal here is to study the $n$-dimensional case.

For any proper domain $G \subset \mathbb{R}^{n}$ we consider the density $\rho(x)=\frac{1}{d(x, \partial G)}, x \in G$. The corresponding metric, denoted by $k_{G}[\mathrm{GP}$, is called the quasihyperbolic metric in $G$. Thus, for $x, y \in G$,

$$
k_{G}(x, y)=\inf _{\gamma} \int_{\gamma} \rho d s
$$

where the infimum is taken over the family of all rectifiable curves $\gamma$ in $G$ joining $x$ to $y$.

Gehring and Palka GP proved the following upper bound for Problem [1.1. Presumably this bound could be improved.

1.7. Theorem (GP, Lemma 3.1]). In Problem $1.1(2)$ we can choose $K(f) \leqslant$ $\exp \left(c_{2} k_{G}(a, b)\right)$, where $c_{2}>0$ only depends on the dimension $n$.

In the case of uniform domains with connected boundary, a lower bound was given by the second author in VU1; see Theorem 3.2 below. For the case of the unit ball this problem was studied by G.D. Anderson and M.K. Vamanamurthy [AV], who found the following counterpart for Theorem 1.3 for dimensions $n \geq 3$. Note, in particular, that here they use the linear dilatation and that an additional symmetry hypothesis is required. They conjectured on p. 2 of [AV] that the result also holds without this additional hypothesis.

1.8. Theorem ( $(\mathrm{AV}])$. For $f \in I d\left(\partial B^{n}\right)$ with the linear dilatation $H(f)=K(c f$. [V, p. 78]) we have

$$
|f(0)| \leqslant c_{1},
$$

where $c_{1}$ is as in (1.4) provided that $f$ satisfies a certain symmetry hypothesis. 
The goal of this paper is to prove the following theorem where no extra symmetry hypotheses are required.

1.9. Theorem. If $f \in I d_{K}\left(\partial B^{n}\right)$, then for all $x \in B^{n}$,

$$
\rho_{B^{n}}(f(x), x) \leqslant \log \frac{1-a}{a}, \quad a=\varphi_{1 / K, n}(1 / \sqrt{2})^{2},
$$

where $\rho_{B^{n}}$ is the hyperbolic metric defined in Lemma 2.1 and $\varphi_{K, n}$ is as in (2.10).

1.10. Theorem. If $f \in I d_{K}\left(\partial B^{n}\right)$, then for all $x \in B^{n}, n \geq 2$, and $K \in[1,17]$

$$
|f(x)-x| \leq \frac{9}{2}(K-1) .
$$

For $n=2$ and $K>1$ we have

$$
|f(x)-x| \leqslant \frac{b}{2}(K-1), \quad b \leqslant 4.38 .
$$

The theory of $K$-quasiregular mappings in $\mathbb{R}^{n}, n \geq 3$, with maximal dilatation $K$ close to 1 has been extensively studied by Yu.G. Reshetnyak $\mathrm{R}$ under the name "stability theory". By Liouville's theorem we expect that when $n \geq 3$ is fixed and $K \rightarrow 1$ the $K$-quasiregular maps "stabilize", become more and more like Möbius transformations, and this is the content of the deep main results of R (see p. 286). We have been unable to decide whether Theorem 1.9 follows from Reshetnyak's stability theory in a simple way. V. I. Semenov [S] has also made significant contributions to this theory. For the plane case, P. P. Belinskii has found several sharp results in $\mathrm{Be}$.

Finally, it seems to be an open problem whether a new kind of stability behavior holds: If $K>1$ is fixed, do maps in $I d_{K}\left(\partial B^{n}\right)$ approach identity when $n \rightarrow \infty$ ? Our results do not answer this question. This kind of behavior is anticipated in [AVV, Open problem 9, p. 478].

\section{Preliminary Results}

We shall follow the terminology of [V], where, for instance, the moduli of curve families are discussed. For the hyperbolic metric $\rho_{B^{n}}$ of the unit ball $B^{n}$ our main reference is [B]. In the next lemma we give the useful estimate (2.3) for it. Some applications of (2.3) were given in [VU2, pp. 141-142]. Very recently, Earle and Harris $[\mathrm{EH}]$ have given several applications and extended this inequality to other metrics such as the Carathéodory metric.

2.1. Lemma. For $x, y \in B^{n}$ let $t=\sqrt{\left(1-|x|^{2}\right)\left(1-|y|^{2}\right)}$. Then

$$
\begin{gathered}
\tanh ^{2} \frac{\rho_{B^{n}}(x, y)}{2}=\frac{|x-y|^{2}}{|x-y|^{2}+t^{2}}, \\
|x-y| \leqslant 2 \tanh \frac{\rho_{B^{n}}(x, y)}{4}=\frac{2|x-y|}{\sqrt{|x-y|^{2}+t^{2}}+t},
\end{gathered}
$$

where equality holds for $x=-y$.

Proof. For (2.2) see [B, p. 40]; for (2.3) see [VU2, (2.18), 2.27]. 
Next, we consider a decreasing homeomorphism $\mu:(0,1) \longrightarrow(0, \infty)$ defined by

$$
\mu(r)=\frac{\pi}{2} \frac{\mathcal{K}\left(r^{\prime}\right)}{\mathcal{K}(r)}, \quad \mathcal{K}(r)=\int_{0}^{1} \frac{d x}{\sqrt{\left(1-x^{2}\right)\left(1-r^{2} x^{2}\right)}},
$$

where $\mathcal{K}(r)$ is Legendre's complete elliptic integral of the first kind and $r^{\prime}=$ $\sqrt{1-r^{2}}$, for all $r \in(0,1)$.

The Hersch-Pfluger distortion function is an increasing homeomorphism $\varphi_{K}$ : $(0,1) \longrightarrow(0,1)$ defined by

$$
\varphi_{K}(r)=\mu^{-1}(\mu(r) / K)
$$

for all $r \in(0,1), K>0$. By continuity we set $\varphi_{K}(0)=0, \varphi_{K}(1)=1$. From (2.4) we see that $\mu(r) \mu\left(r^{\prime}\right)=\left(\frac{\pi}{2}\right)^{2}$ and from this we are able to conclude a number of properties of $\varphi_{K}$. For instance, by [AVV, Thm 10.5, p. 204],

$$
\varphi_{K}(r)^{2}+\varphi_{1 / K}\left(r^{\prime}\right)^{2}=1, \quad r^{\prime}=\sqrt{1-r^{2}},
$$

holds for all $K>0, r \in(0,1)$.

2.7. Proof of Lemma 1.6. By [AVV, (5.27)] we have for $y>0$,

$$
\sqrt{1-\tanh ^{2} y}<\sqrt{1-\tanh ^{8} y}<\mu^{-1}(y)<4 e^{-y} .
$$

With

this inequality yields

$$
y=\log \frac{\sqrt{K}+1}{\sqrt{K}-1}=2 \operatorname{artanh}(1 / \sqrt{K})
$$

$$
\frac{\sqrt{K}-1}{K+1}<c_{1}=\mu^{-1}(y)<4 \frac{\sqrt{K}-1}{\sqrt{K}+1}<2 \frac{K-1}{\sqrt{K}+1} .
$$

2.8. The Grötzsch and Teichmüller rings. The Grötzsch and Teichmüller ring domains $R_{G}(s), s>1$, and $R_{T}(t), t>0$, are doubly connected domains with complementary components $\left(\bar{B}^{n},\left[s e_{1}, \infty\right)\right)$ and $\left(\left[-e_{1}, 0\right],\left[t e_{1}, \infty\right)\right)$, respectively. Their capacities $\operatorname{cap} R_{G}(s)$ and $\operatorname{cap} R_{T}(t)$ are often used below. The Grötzsch capacity $\gamma_{n}(s)=\operatorname{cap}_{G}(s)$ is a decreasing homeomorphism $\gamma_{n}:(1, \infty) \longrightarrow(0, \infty)$; see VU2, p. 66] and [AVV, Section 8]. The Teichmüller capacity $\tau_{n}(t)=\operatorname{cap} R_{T}(t)$, is a decreasing homeomorphism $\tau_{n}:(0, \infty) \rightarrow(0, \infty)$ connected with $\gamma_{n}$ by the identity

$$
\tau_{n}(t)=2^{1-n} \gamma_{n}(\sqrt{1+t}), t>0 .
$$

Given $E, F, G \subset \mathbb{R}^{n}$ we use the notation $\Delta(E, F ; G)$ for the family of all curves that join the sets $E$ and $F$ in $G$ and $M(\Delta(E, F ; G))$ for its modulus; see $\mathrm{V}$, Chapter I]. Then $\tau_{n}(t)=M\left(\Delta\left(E, F ; \mathbb{R}^{n}\right)\right)$ where $E$ and $F$ are the complementary components of the Teichmüller ring and a similar relation also holds for $\gamma_{n}(s)$.

We use the standard notation

$$
\varphi_{K, n}(r)=\frac{1}{\gamma_{n}^{-1}\left(K \gamma_{n}(1 / r)\right)} .
$$

Then $\varphi_{K, n}:(0,1) \longrightarrow(0,1)$ is an increasing homeomorphism; see VU2, (7.44)]. Because $\gamma_{2}(1 / r)=2 \pi / \mu(r)$ by [VU2, (5.56)] and [LV], it follows that $\varphi_{K, 2}(r)$ is the same as the function $\varphi_{K}(r)$ in (2.5). 
2.11. The key constant. The special functions introduced above will have a crucial role in what follows. For the sake of easy reference we give here some wellknown identities between them that can be found in AVV]. First, the function

$$
\eta_{K, n}(t)=\tau_{n}^{-1}\left(\tau_{n}(t) / K\right)=\frac{1-\varphi_{1 / K, n}(1 / \sqrt{1+t})^{2}}{\varphi_{1 / K, n}(1 / \sqrt{1+t})^{2}}, K>0
$$

defines an increasing homeomorphism $\eta_{K, n}:(0, \infty) \rightarrow(0, \infty)$ (cf. AVV, p. 193]). The constant $(1-a) / a, a=\varphi_{1 / K, n}(1 / \sqrt{2})^{2}$, in Theorem 1.9 can be expressed as follows for $K>1$ :

$$
(1-a) / a=\eta_{K, n}(1)=\tau_{n}^{-1}\left(\tau_{n}(1) / K\right) .
$$

Furthermore, by (2.6),

$$
\eta_{K, 2}(t)=\frac{s^{2}}{1-s^{2}}, \quad s=\varphi_{K, 2}(\sqrt{t /(1+t)})
$$

and

$$
\eta_{K, 2}(1) \in\left(e^{\pi(K-1)}, e^{b(K-1)}\right)
$$

where $b=(4 / \pi) \mathcal{K}(1 / \sqrt{2})^{2}=4.376879 \ldots$. Note that the constant $\lambda(K)$ in [AVV, 10.33] is the same as $\eta_{K, 2}(1)$. In passing we remark that P. P. Belinskii in Be, Lemma 12, p. 80] gave the inequality

$$
\eta_{K, 2}(1) \equiv \lambda(K)<1+12(K-1)
$$

for $K$ close to 1 , however, with an incorrect proof as pointed out in $\mathrm{AQVu}$. (3.10)]. Because this inequality is one of the key technical estimates of $\mathrm{Be}$, it is fortunate that this error was detected and a correct proof was later found (see $\mathrm{AQVu}$, Corollary 3.7]).

For the proof of Lemma 2.24, we record a lower bound for $\varphi_{1 / K, n}(r)$. The constant $\lambda_{n} \in\left[4,2 e^{n-1}\right)$ is the so-called Grötzsch ring constant; see [AVV].

2.16. Lemma ([VU2, 7.47, 7.50]). For $n \geq 2, K \geq 1$, and $0 \leq r \leq 1$,

$$
\begin{gathered}
\varphi_{1 / K, n}(r) \geq \lambda_{n}^{1-\beta} r^{\beta}, \beta=K^{1 /(n-1)}, \\
\lambda_{n}^{1-\beta} \geq 2^{1-\beta} K^{-\beta} \geq 2^{1-K} K^{-K} .
\end{gathered}
$$

In the next lemma we consider two strictly increasing continuous functions $p, q$ : $[1, \infty) \rightarrow(0, \infty)$ such that $p(1)<q(1)$ and that the opposite inequality $p\left(x_{1}\right)>$ $q\left(x_{1}\right)$ holds for some $x_{1}>1$. In the first part of the lemma we find, for the given functions, a concrete value $\varepsilon>0$ such that $p(x)<q(x)$ for all $x \in[1,1+\varepsilon)$. In the second part of the lemma we apply an iterative method with $1+\varepsilon$ as a starting value to find the largest number $a \in\left[1+\varepsilon, x_{1}\right)$ such that $p(x)<q(x)$ for all $x \in[1, a)$ and show that $a>17$.

2.19. Lemma. (1) For all $m, n \geqslant 1$ there is $M>1$ such that the inequality

$$
\log \left(2^{m x-m+1} x^{n x}-1\right) \leqslant(2 m \log 2+2 n)(x-1)
$$

holds for $x \in[1, M]$ with equality only for $x=1$. Moreover, with $t=(m \log 2-$ $n) /(2 n), M$ can be chosen as

$$
M=\sqrt{\frac{(m-1) \log 2+\log \left(1+\frac{(n+m \log 2)^{2}}{n}\right)}{n}+t^{2}}-t .
$$


(2) Let $p(x)=\log \left(2^{m x-m+1} x^{n x}-1\right), q(x)=(2 m \log 2+2 n)(x-1)$ and let us use the above notation. Let $a_{0}=M$ and $a_{n+1}=p^{-1}\left(q\left(a_{n}\right)\right)$ for $n \geqslant 1$. Then the sequence $a_{n}$ is increasing and bounded. If $a=\lim _{n \rightarrow \infty} a_{n}$, then the inequality (2.20) holds for $x \in[1, a]$ with equality iff $x \in\{1, a\}$. For $m=3$ and $n=2$ we have $a>17$.

Proof. Let

$u(x)=(m x-m+1) \log 2+n x \log x, \quad v(x)=\log \left(e^{u(x)}-1\right)=\log \left(2^{m x-m+1} x^{n x}-1\right)$.

Then we have

$$
\begin{aligned}
v^{\prime \prime}(x) & =\left(\log \left(e^{u(x)}-1\right)\right)^{\prime \prime}=\left(\frac{u^{\prime}(x) e^{u(x)}}{e^{u(x)}-1}\right)^{\prime} \\
& =\frac{\left(u^{\prime \prime}(x) e^{u(x)}+\left(u^{\prime}(x)\right)^{2} e^{u(x)}\right)\left(e^{u(x)}-1\right)-\left(u^{\prime}(x) e^{u(x)}\right)^{2}}{\left(e^{u(x)}-1\right)^{2}} \\
& =\frac{e^{u(x)}}{\left(e^{u(x)}-1\right)^{2}} \cdot\left(\left(u^{\prime \prime}(x)+\left(u^{\prime}(x)\right)^{2}\right)\left(e^{u(x)}-1\right)-\left(u^{\prime}(x)\right)^{2} e^{u(x)}\right) \\
& =\frac{e^{u(x)}}{\left(e^{u(x)}-1\right)^{2}} \cdot\left(u^{\prime \prime}(x)\left(e^{u(x)}-1\right)-\left(u^{\prime}(x)\right)^{2}\right) .
\end{aligned}
$$

Thus,

$$
v^{\prime \prime}(x) \leqslant 0 \quad \Leftrightarrow \quad u^{\prime \prime}(x)\left(e^{u(x)}-1\right) \leqslant\left(u^{\prime}(x)\right)^{2} .
$$

Since

$$
e^{u(x)}=2^{m x-m+1} x^{n x}, \quad u^{\prime}(x)=n+m \log 2+n \log x, \quad u^{\prime \prime}(x)=\frac{n}{x},
$$

we have

$$
v^{\prime \prime}(x) \leqslant 0 \Leftrightarrow \frac{n}{x}\left(2^{m x-m+1} x^{n x}-1\right) \leqslant(n+m \log 2+n \log x)^{2} .
$$

Therefore we see that for $x \geqslant 1$ the condition $v^{\prime \prime}(x) \leqslant 0$ is equivalent to

$$
2^{m x-m+1} x^{n x}-1 \leqslant \frac{x}{n}(n+m \log 2+n \log x)^{2} .
$$

Let $f(x)=2^{m x-m+1} x^{n x}-1$ and $g(x)=\frac{x}{n}(n+m \log 2+n \log x)^{2}$. Both functions $f$ and $g$ are increasing on $[1,+\infty)$ and $f(1)<g(1)$ because

$$
f(1)=1 \leqslant n=\frac{1}{n} \cdot n^{2}<\frac{1}{n}(n+m \log 2)^{2}=g(1) .
$$

By the continuity of $f$ we can conclude that there is $M>1$ such that $f(M) \leqslant g(1)$. For such $M$, we have

$$
f(x) \leqslant f(M) \leqslant g(1) \leqslant g(x), \quad x \in[1, M]
$$

This implies that $v$ is concave on $[1, M]$ and therefore

$$
v(x) \leqslant v(1)+v^{\prime}(1)(x-1), \quad x \in[1, M],
$$

i.e.,

$$
\log \left(2^{m x-m+1} x^{n x}-1\right) \leqslant(2 m \log 2+2 n)(x-1), \quad x \in[1, M] .
$$

The inequality $f(x) \leqslant g(1)$ is equivalent to

$$
(m x-m+1) \log 2+n x \log x \leqslant \log \left(1+\frac{(n+m \log 2)^{2}}{n}\right) .
$$


Because

$$
(m x-m+1) \log 2+n x \log x \leqslant(m x-m+1) \log 2+n x(x-1),
$$

the inequality (2.21) is a consequence of the inequality

$$
(m x-m+1) \log 2+n x(x-1) \leqslant \log \left(1+\frac{(n+m \log 2)^{2}}{n}\right) .
$$

In (2.22) the equality sign holds only for $x=1$. Because

$$
1+\frac{(n+m \log 2)^{2}}{n}>1+\frac{n^{2}}{n}=1+n \geqslant 2,
$$

the inequality (2.23) is a strict inequality for $x=1$. By this reasoning, the greater root of the quadratic equation

$$
(m x-m+1) \log 2+n x(x-1)=\log \left(1+\frac{(n+m \log 2)^{2}}{n}\right)
$$

is greater than 1. If we denote this root with $M$, the inequality (2.21) holds for $x \in[1, M]$ with equality only for $x=1$. The first part of the lemma is proved.

Now we prove the second part of the inequality. Both of the functions $p(x)$ and $q(x)$ are continuous and increasing. Consequently, $r(x)=p^{-1}(x)$ is continuous and increasing. Because

$$
p\left(a_{1}\right)=q\left(a_{0}\right)>p\left(a_{0}\right),
$$

using monotonicity of $p(x)$, we can conclude that $a_{1}>a_{0}$. Now, by induction and monotonicity of $r$, we can conclude that the sequence $a_{n}$ is increasing. Now for $x \in\left[a_{n}, a_{n+1}\right)$ we have

$$
p(x)<p\left(a_{n+1}\right)=q\left(a_{n}\right) \leqslant q(x) .
$$

Therefore the inequality $p(x)<q(x)$ holds for $x \in \bigcup_{n=0}^{\infty}\left[a_{n}, a_{n+1}\right)=\left[a_{0}, a\right)$ and, using what was already proved, we see that the inequality $p(x)<q(x)$ holds for the whole interval $1<x<a$. For $x \geqslant 1$ we see that $m x-m+1>1$ and $x^{n x} \geqslant 1$ and, consequently,

$$
p(x)=\log \left(2^{m x-m+1} x^{n x}-1\right)>\log \left(2 x^{n x}-1\right) \geqslant n x \log x .
$$

Because $p(x)>n x \log x \geqslant(n \log x)(x-1)$ the inequality $p(c)>q(c)$ holds for $c$ such that $n \log c \geqslant 2 m \log 2+2 n$. It is easy to check that it is true for $c=2^{\frac{2 m}{n}} e^{2}$. It implies that $a$ is finite (for example $a<2^{\frac{2 m}{n}} e^{2}$ ) and $a_{n}$ is bounded. The relation $p\left(a_{n+1}\right)=q\left(a_{n}\right)$ and the continuity of both functions show that $\lim p\left(a_{n+1}\right)=$ $p(a)=q(a)=\lim q\left(a_{n}\right)$. The lower bound for $a$ follows because $a_{36}>17$.

2.24. Lemma. If $a=\varphi_{1 / K, n}(1 / \sqrt{2})^{2}$ is as in Theorem 1.9, then for $M>1$ and $\beta \in[1, M]$,

$$
\log \left(\frac{1-a}{a}\right) \leq \log \left(\lambda_{n}^{2(\beta-1)} 2^{\beta}-1\right) \leq V(n)(\beta-1)
$$

with $V(n)=\left(2 \log \left(2 \lambda_{n}^{2}\right)\right)\left(2 \lambda_{n}^{2}\right)^{M-1}$ and for $K \in[1,17]$,

$$
\log \left(\frac{1-a}{a}\right) \leqslant(K-1)(4+6 \log 2)<9(K-1),
$$


with equality only for $K=1$. For $n=2$ and $K>1$,

$$
\log \left(\frac{1-a}{a}\right)=\log \left(\frac{\varphi_{K, 2}(1 / \sqrt{2})^{2}}{\varphi_{1 / K, 2}(1 / \sqrt{2})^{2}}\right) \leqslant b(K-1),
$$

where $b=(4 / \pi) \mathcal{K}(1 / \sqrt{2})^{2} \leq 4.38$.

Proof. For $\beta \in[1, M]$ we have by (2.17),

$$
\log \left(\frac{1-a}{a}\right) \leq \log \left(\lambda_{n}^{2(\beta-1)} 2^{\beta}-1\right) .
$$

Furthermore, we have

$$
\frac{\log \left(\lambda_{n}^{2(\beta-1)} 2^{\beta}-1\right)}{\beta-1} \leqslant 2 \frac{\left(2 \lambda_{n}^{2}\right)^{\beta-1}-1}{\beta-1} \leqslant\left(2 \log \left(2 \lambda_{n}^{2}\right)\right)\left(2 \lambda_{n}^{2}\right)^{M-1} .
$$

The second inequality follows from the inequality $\log (t) \leqslant t-1$ and the third one from Lagrange's theorem and the monotonicity of the function $\left(2 \log \left(2 \lambda_{n}^{2}\right)\right)\left(2 \lambda_{n}^{2}\right)^{x-1}$. This proves (2.25).

From (2.18) it follows that the constant $a$ satisfies the inequality

$$
a \geq 2^{2(1-K)} K^{-2 K}(1 / \sqrt{2})^{2 K}
$$

and also

$$
1 / a \leq 2^{3 K-2} K^{2 K}, \quad K>1 .
$$

By Lemma 2.19 we have

$$
\log \left(2^{3 K-2} K^{2 K}-1\right) \leqslant(4+6 \log 2)(K-1)
$$

for $K \in[1,17]$ with equality only for $K=1$. Now, from

$$
\frac{1-a}{a}<2^{3 K-2} K^{2 K}-1, \quad K>1,
$$

we conclude that

$$
\log \left(\frac{1-a}{a}\right) \leqslant(4+6 \log 2)(K-1)<9(K-1) .
$$

For the case $n=2$ we can apply the identity (2.14) and the inequality in (2.15).

\section{Proof of Theorem 1.9}

Lemma 3.1 and Theorem 3.2 deal with the first part of Problem 1.1 .

3.1. Lemma ([VU1]). Let $f \in I d_{K}(\partial G), a, b \in G, f(a)=b$ and let the boundary $\partial G$ be connected. If $x \in \partial G$ is such that $d(a)=d(a, \partial G)=|a-x| \leqslant|b-x|$, then

$$
K(f) \geqslant \bar{d}_{n}\left(\log \frac{|b-x|}{|a-x|}\right)^{n}, \quad \bar{d}_{n}=\frac{c_{n}}{\omega_{n-1}} \frac{(n-1)^{n-1}}{n^{n}} .
$$

The following result was proved in [VU1, however, under the condition that the points are far away from each other. The general case follows from the original result by reducing the constant. In VU1, an example was given to the effect that Theorem 3.2 cannot be improved to the claim that $a, b \in G, k_{G}(a, b)>0$ implies $K(f)>1$. 
3.2. Theorem (U1]). Let $f \in I d_{K}(\partial G), a, b \in G$ with $f(a)=b$. If $G$ is $a$ uniform domain with connected boundary $\partial G$, then

$$
K(f) \geqslant d_{n} k_{G}(a, b)^{n}
$$

where $d_{n}$ depends only on $n$ and $G$.

3.3. Proof of Theorem 1.9, Fix $x \in B^{n}$ and let $T_{x}$ denote a Möbius transformation of $\overline{\mathbb{R}^{n}}$ with $T_{x}\left(B^{n}\right)=B^{n}$ and $T_{x}(x)=0$. Define $g: \mathbb{R}^{n} \longrightarrow \mathbb{R}^{n}$ by setting $g(z)=T_{x} \circ f \circ T_{x}^{-1}(z)$ for $z \in B^{n}$ and $g(z)=z$ for $z \in \mathbb{R}^{n} \backslash B^{n}$. Then $g \in I d_{K}\left(\partial B^{n}\right)$ with $g(0)=T_{x}(f(x))$. By the invariance of $\rho_{B^{n}}$ under the group $\mathcal{G M}\left(B^{n}\right)$ of Möbius selfautomorphisms of $B^{n}$ we see that for $x \in B^{n}$,

$$
\rho_{B^{n}}(f(x), x)=\rho_{B^{n}}\left(T_{x}(f(x)), T_{x}(x)\right)=\rho_{B^{n}}(g(0), 0) .
$$

Choose $z \in \partial B^{n}$ such that $g(0) \in[0, z]=\{t z: 0 \leqslant t \leqslant 1\}$. Let $E^{\prime}=\{-s z$ : $s \geqslant 1\}, \Gamma^{\prime}=\Delta\left([g(0), z], E^{\prime} ; \mathbb{R}^{n}\right)$ and $\Gamma=\Delta\left(g^{-1}[g(0), z], g^{-1} E^{\prime} ; \mathbb{R}^{n}\right)$. Observe that $E^{\prime}=g^{-1} E^{\prime}$ because $g \in I d_{K}\left(\partial B^{n}\right)$.

The spherical symmetrization with center at 0 yields, by [AVV] Thm 8.44],

$$
M(\Gamma) \geqslant \tau_{n}(1) \quad\left(=2^{1-n} \gamma_{n}(\sqrt{2})\right)
$$

because $g(x)=x$ for $x \in \mathbb{R}^{n} \backslash B^{n}$. Next, we see by the choice of $\Gamma^{\prime}$ that

$$
M\left(\Gamma^{\prime}\right)=\tau_{n}\left(\frac{1+|g(0)|}{1-|g(0)|}\right) .
$$

By $K$-quasiconformality we have $M(\Gamma) \leqslant K M\left(\Gamma^{\prime}\right)$ implying

$$
\exp \left(\rho_{B^{n}}(0, g(0))\right)=\frac{1+|g(0)|}{1-|g(0)|} \leqslant \tau_{n}^{-1}\left(\tau_{n}(1) / K\right)=\frac{1-a}{a} .
$$

The last equality follows from (2.13). Finally, (3.4) and (3.5) complete the proof.

3.6. Proof of Theorem 1.10] We have

$$
\begin{aligned}
|f(x)-x| & \leqslant 2 \tanh \left(\frac{\rho_{B^{n}}(f(x), x)}{4}\right) \leqslant 2 \tanh \left(\frac{\log \left(\frac{1-a}{a}\right)}{4}\right) \\
& \leqslant 2 \tanh \left(\frac{(K-1)(4+6 \log 2)}{4}\right) \\
& \leqslant(K-1)(2+3 \log 2) \leqslant \frac{9}{2}(K-1) .
\end{aligned}
$$

The first inequality follows from (2.3), the second one from Theorem 1.9, the third one from Lemma 2.24 and the fourth one from the inequality $\tanh (t) \leqslant t$ for $t \geqslant 0$.

For $n=2$ we use the same first two steps and the planar case of Lemma 2.24 to derive the inequality

$$
|f(x)-x| \leqslant \frac{b}{2}(K-1) .
$$

A lower bound corresponding to the upper bound in (1.11) is given in the next lemma. 
3.7. Lemma. For $f \in \operatorname{Id}(\partial G)$ let

$$
\delta(f) \equiv \sup \{|f(z)-z|: z \in G\} .
$$

Then for $f \in I d_{K}\left(\partial B^{n}\right), K>1, \alpha=K^{1 /(1-n)}$,

$$
\delta(f) \geq(1-\alpha) \alpha^{\alpha /(1-\alpha)}>\frac{1}{e}(1-\alpha) .
$$

Proof. The radial stretching $f: B^{n} \rightarrow B^{n}, n \geq 2$, defined by $f(z)=|z|^{\alpha-1} z, z \in$ $B^{n},(0<\alpha<1)$, is $K$-quasiconformal with $\alpha=K^{1 /(1-n)}$ [V] p. 49] and $f \in$ $I d_{K}\left(\partial B^{n}\right)$. Now we have

$$
|f(z)-z|=\left.|| z\right|^{\alpha-1} z-z|=| r^{\alpha}-r|, \quad| z \mid=r .
$$

Furthermore, we see that

$$
\delta(f)=\sup _{0<r<1}\left(r^{\alpha}-r\right),
$$

where the supremum is attained for $r=r_{\alpha}=\left(\frac{1}{\alpha}\right)^{\frac{1}{\alpha-1}}$, so

$$
\delta(f)=(1-\alpha) \alpha^{\alpha /(1-\alpha)} .
$$

A crude, but simple, estimate is

$$
\delta(f) \geq(1 / e)^{\alpha}-(1 / e)=\frac{1}{e}\left(\frac{1}{e^{\alpha-1}}-1\right)=\frac{1}{e}\left(e^{1-\alpha}-1\right) \geqslant \frac{1}{e}(1-\alpha) .
$$

3.9. Theorem. Let $f: \overline{\mathbb{R}^{n}} \longrightarrow \overline{\mathbb{R}^{n}}$ be a $K$-quasiconformal homeomorphism with $f(\infty)=\infty$ and $B^{n}(m) \subset f\left(B^{n}\right) \subset B^{n}(M)$, where $0<m \leq 1 \leq M$. Then

$$
\eta_{1 / K, n}\left(\frac{1+|x|}{1-|x|}\right) \leqslant \frac{M+|f(x)|}{m-|f(x)|}
$$

and

$$
\frac{m+|f(x)|}{M-|f(x)|} \leqslant \eta_{K, n}\left(\frac{1+|x|}{1-|x|}\right)
$$

for all $x \in B^{n}$ where $\eta_{K, n}(t)=\tau_{n}^{-1}\left(\tau_{n}(t) / K\right)$.

In particular, if $m=1=M$, then we have

$$
\eta_{1 / K, n}\left(\frac{1+|x|}{1-|x|}\right) \leqslant \frac{1+|f(x)|}{1-|f(x)|} \leqslant \eta_{K, n}\left(\frac{1+|x|}{1-|x|}\right) .
$$

Proof. The proof is similar to the proof of Theorem 1.9. Fix $x \in B^{n}$ and choose $z^{\prime} \in \partial f\left(B^{n}\right)$ such that $f(x) \in\left[0, z^{\prime}\right]$ and $\left[f(x), z^{\prime}\right) \subset f\left(B^{n}\right)$ and fix $z^{\prime \prime} \in \partial f\left(B^{n}\right)$ such that $z^{\prime}, 0, z^{\prime \prime}$ are on the same line, $0 \in\left[z^{\prime}, z^{\prime \prime}\right]$, and $\left\{-s z^{\prime \prime}: s \geqslant 1\right\} \subset$ $\mathbb{R}^{n} \backslash f\left(B^{n}\right)$. Let $\Gamma^{\prime}=\Delta\left(\left[f(x), z^{\prime}\right], E^{\prime} ; \mathbb{R}^{n}\right), E^{\prime}=\left\{-s z^{\prime \prime}: s \geqslant 1\right\}$ and $\Gamma=$ $\Delta\left(f^{-1}\left[f(x), z^{\prime}\right], f^{-1} E^{\prime} ; \mathbb{R}^{n}\right)$. Then

$$
M\left(\Gamma^{\prime}\right) \leq \tau_{n}\left(\frac{m+|f(x)|}{M-|f(x)|}\right)
$$

while applying a spherical symmetrization with center at the origin gives

$$
M(\Gamma) \geqslant \tau_{n}\left(\frac{1+|x|}{1-|x|}\right)
$$


because $f^{-1} E^{\prime}$ connects $\partial B^{n}$ and $\infty$. Then the inequality $M(\Gamma) \leqslant K M\left(\Gamma^{\prime}\right)$ yields

$$
\begin{gathered}
\tau_{n}\left(\frac{1+|x|}{1-|x|}\right) \leq K \tau_{n}\left(\frac{m+|f(x)|}{M-|f(x)|}\right), \\
\tau_{n}^{-1}\left(\frac{1}{K} \tau_{n}\left(\frac{1+|x|}{1-|x|}\right)\right) \geq \frac{m+|f(x)|}{M-|f(x)|}, \\
\frac{m+|f(x)|}{M-|f(x)|} \leqslant \eta_{K, n}\left(\frac{1+|x|}{1-|x|}\right) .
\end{gathered}
$$

The lower bound follows if we apply a similar argument to $f^{-1}$ and the lower bound

$$
M\left(\Gamma^{\prime}\right) \geq \tau_{n}\left(\frac{M+|f(x)|}{m-|f(x)|}\right) .
$$

3.11. Remark. Putting $x=0, m=1=M$ in (3) we obtain by (2.13) for a $K$ quasiconformal homeomorphism $f: \overline{\mathbb{R}^{n}} \longrightarrow \overline{\mathbb{R}^{n}}$ with $f(\infty)=\infty$ and $f\left(B^{n}\right)=B^{n}$ that

$$
|f(0)| \leq 1-2 a, a=\varphi_{1 / K, n}(1 / \sqrt{2})^{2} .
$$

Furthermore, if we use the lower bound (2.18) from Lemma 2.16 we obtain

$$
|f(0)| \leq 1-2^{1-\beta} 4^{1-K} K^{-2 K} .
$$

In the special case when $n=2$ we have

$$
|f(0)| \leq 1-2^{3(1-K)} K^{-2 K} \leq(2+3 \log 2)(K-1) .
$$

Note that this last inequality does not suppose that $f \in I d_{K}\left(\partial B^{n}\right)$, only the hypotheses of Theorem 3.9 are needed.

3.12. Corollary. Let $n=2$ in addition to the hypotheses of Theorem 3.9. Then

$$
\eta_{K, 2}(t)=\frac{u^{2}}{1-u^{2}}=\frac{u^{2}}{v^{2}},
$$

where $u=\varphi_{K, 2}\left(\sqrt{\frac{t}{1+t}}\right), v=\varphi_{1 / K, 2}\left(\frac{1}{\sqrt{1+t}}\right)$ and

$$
|f(x)| \leqslant 2 \varphi_{K, 2}\left(\sqrt{\frac{1+|x|}{2}}\right)^{2}-1
$$

for all $x \in B^{2}$.

Proof. The identity (3.13) holds by (2.14). Next Theorem 3.9 together with (3.13) yield

$$
\frac{1+|f(x)|}{1-|f(x)|} \leqslant \frac{w^{2}}{1-w^{2}}
$$

where $w=\varphi_{K, 2}\left(\sqrt{\frac{1+|x|}{2}}\right)$. Solving this for $|f(x)|$ yields (3.14). 
3.15. Remark. By the $K$-quasiconformal Schwarz lemma, if $f: B^{2} \longrightarrow B^{2}$ is $K$ quasiconformal with $f(0)=0$, then $|f(z)| \leqslant \varphi_{K, 2}(|z|)$, for all $z \in B^{2}$, where the sharp bound is attained for a map with $f\left(B^{2}\right)=B^{2}([\overline{\mathrm{LV}}])$. Note that in Corollary 3.12 the condition $f(0)=0$ is not required. We conclude that

$$
\varphi_{K, 2}(r) \leqslant 2 \varphi_{K, 2}\left(\sqrt{\frac{1+r}{2}}\right)^{2}-1 .
$$

Writing $A(r, s)=\sqrt{\frac{r+s}{2}}(3.16)$ says that if $t=1, r \in(0,1)$, then

$$
A\left(\varphi_{K, 2}(t), \varphi_{K, 2}(r)\right) \leqslant \varphi_{K, 2}(A(t, r)) .
$$

It seems natural to expect that this inequality holds for all $t, r \in(0,1)$.

\section{ACKNOWLEDGEMENT}

The research of the second author was supported by the project "Quasiconformal Maps" nr 209539 of Matti Vuorinen funded by the Academy of Finland.

\section{REFERENCES}

[AV] G.D. Anderson and M.K. Vamanamurthy: An extremal displacement mapping in $n$ space. Complex analysis Joensuu 1978 (Proc. Colloq., Univ. Joensuu, Joensuu, 1978), pp. 1-9, Lecture Notes in Math., 747, Springer, Berlin, 1979. MR553026 (81b:30030)

[AQVu] G.D. Anderson, S.-L. Qiu, and M. Vuorinen: Modular Equations and Distortion Functions. Ramanujan J. 18 (2009), no. 2, 147-169. MR2475934 (2010a:30032)

[AVV] G.D. Anderson, M.K. Vamanamurthy, and M.K. Vuorinen: Conformal invariants, inequalities, and quasiconformal maps. John Wiley \& Sons, Inc., New York, 1997. MR.1462077 (98h:30033)

[B] A.F. BeARdon: The geometry of discrete groups. Springer-Verlag, New York, 1983. MR698777 (85d:22026)

[Be] P.P. BelinskiI: General properties of quasiconformal mappings (Russian). Izd. Nauka, Novosibirsk, 1974. MR0407275 (53:11054)

[EH] C.J. EARle ANd L.A. HARRIS: Inequalities for the Carathéodory and Poincaré metrics in open unit balls, Manuscript, 2008.

[GP] F.W. Gehring And B.P. PALKa: Quasiconformally homogeneous domains. J. Analyse Math. 30 (1976), 172-199. MR0437753 (55:10676)

[Kr] S.L. KRUSHKaL: Variational principles in the theory of quasiconformal maps. Handbook of complex analysis: Geometric function theory. Edited by R. Kuhnau. Vol. 2, 31-98, Elsevier, Amsterdam, 2005. MR2121857 (2005k:30039a)

[K] J. Krzyż: On an extremal problem of F. W. Gehring. Bull. Acad. Polon. Sci. Sér. Sci. Math. Astronom. Phys. 16, 1968, 99-101. MR0226002 (37:1592)

[LV] O. Lehto and K.I. Virtanen: Quasiconformal mappings in the plane. Second edition. Translated from the German by K.W. Lucas. Die Grundlehren der mathematischen Wissenschaften, Band 126. Springer-Verlag, New York, Heidelberg, 1973. viii+258 pp. MR0344463 (49:9202)

[M] G.J. MARTIN: The distortion theorem for quasiconformal mappings, Schottky's theorem and holomorphic motions. Proc. Amer. Math. Soc. 125 (1997), no. 4, 1095-1103. MR1363178 (97g:30017)

[R] Yu.G. Reshetnyak: Stability theorems in geometry and analysis. Translated from the 1982 Russian original by N.S. Dairbekov and V.N. Dyatlov, and revised by the author. Translation edited and with a foreword by S.S. Kutateladze. Mathematics and its Applications, 304. Kluwer Academic Publishers Group, Dordrecht, 1994. xii+394 pp. ISBN: 0-7923-3118-4. MR1326375 (96i:30016)

[S] V.I. Semenov: Estimates of stability, distortion theorems, and topological properties of quasiregular mappings. Mat. Zametki 51 (1992), 109-113. MR1186539 (93h:30032)

[SV] A.Yu. Solynin And M. VuORInen: Estimates for the hyperbolic metric of the punctured plane and applications. Israel J. Math. 124 (2001), 29-60. MR.1856503(2002j:30071) 
[T] O. Teichmüller: Ein Verschiebungssatz der quasikonformen Abbildung. (German) Deutsche Math. 7, (1944). 336-343. see also Teichmüller, Oswald Gesammelte Abhandlungen. (German) [Collected papers] Edited and with a preface by Lars V. Ahlfors and Frederick W. Gehring. Springer-Verlag, Berlin, New York, 1982. viii+751 pp. MR0018761 $(8: 327 \mathrm{~b})$

[V] J. VÄIsÄLÄ: Lectures on $n$-dimensional quasiconformal mappings. Lecture Notes in Mathematics, Vol. 229. Springer-Verlag, Berlin, New York, 1971. MR0454009 (56:12260)

[VU1] M. Vuorinen: A remark on the maximal dilatation of a quasiconformal mapping. Proc. Amer. Math. Soc. 92(1984), no. 4, 505-508. MR760934 (86a:30039)

[VU2] M. VUORINEn: Conformal geometry and quasiregular mappings. Lecture Notes in Mathematics, 1319. Springer-Verlag, Berlin, 1988. xx+209 pp. MR950174 (89k:30021)

[XC] H. Xinzhong and N.E. ChO: On the distortion theorem for quasiconformal mappings with fixed boundary values. (English summary) J. Math. Anal. Appl. 256 (2001), no. 2, 694-697. MR1821766 (2002a:30030)

Department of Mathematics, Faculty of Organizational Sciences, University of BelGrade, Jove Ilica 154, 11000 Belgrade, Serbia

E-mail address: vesnam@fon.rs

Department of Mathematics, University of Turku, Fin-20014 Turku, Finland

E-mail address: vuorinen@utu.fi 